\title{
Modeling and Simulation of Heat Transfer Phenomenon from Infant Radiant Warmer for a Newborn Baby
}

\author{
Kollol Dey', Ujjwal Kumar Deb ${ }^{2 *}$ \\ ${ }^{1}$ Department of Mathematics, Premier University, Chattogram, Bangladesh \\ ${ }^{2}$ Department of Mathematics, Chittagong University of Engineering and Technology, Chattogram, Bangladesh \\ Email: kolloldey85@gmail.com, ^ukdebmath@cuet.ac.bd
}

How to cite this paper: Dey, K. and Deb, U.K. (2021) Modeling and Simulation of Heat Transfer Phenomenon from Infant Radiant Warmer for a Newborn Baby. Open Journal of Modelling and Simulation, 9, 111-123.

https://doi.org/10.4236/ojmsi.2021.92007

Received: January 16, 2021

Accepted: April 10, 2021

Published: April 13, 2021

Copyright ( $\odot 2021$ by author(s) and Scientific Research Publishing Inc. This work is licensed under the Creative Commons Attribution International License (CC BY 4.0).

http://creativecommons.org/licenses/by/4.0/

\begin{abstract}
Nowadays death of a newborn baby due to hypothermia is one of the vital factors. To overcome the problem infant radiant warmer has been used in hospitals which helps to prevent excessive heat loss of the baby by maintaining a proper temperature. However, in practice, it is critical to regulating proper thermal stability that is exactly required for a premature baby to conquer the heat loss problem. In this study, we have established a computational model for heat transfer analysis using the Finite Element Method. The heat transfer to the surrounding area skin of newborn with the help of Infant Radiant Warmer (IRW) is simulated to study the best range of light source to overcome the hypothermia. We simulate the efficiency and effect of the infant radiant of the thermal radiation using COMSOL Multiphysics program. For this simulation, we considered the distance between the infant's mattress and the bottom surface of the heater unit as $70 \mathrm{~cm}$ and the heater power 500 watts, and 600 watts. We have investigated mattress temperature, baby temperature and surface radiosity which are important to understand to increase newborn baby body temperature. It is found that the temperature of mattress raises up to 36 - 36.5 degrees Celsius and skin temperature raises up to 37 37.5 degrees Celsius.
\end{abstract}

\section{Keywords}

Infant Radiant Warmer, Hypothermia, Modal Analysis, Heat Transfer, FEM

\section{Introduction}

Hypothermia is a potentially dangerous drop in body temperature of new the born babies that makes to be complication [1]. The protecting hypothermia in 
the newborn is important because this hypothermia will repeat the newborn that had any disease to have many terrible until dying according to the high death rate of around 25\% - 50\% [2]. The World Health Organization (WHO) has listed hypothermia as one of the "top killers" during the neonatal period [3]. The symptoms of Hypothermia for infants is an accurate axillary (armpit) or rectal temperature below $36.4^{\circ} \mathrm{C}\left(97.5^{\circ} \mathrm{F}\right)$, a weak cry, low energy level, lethargic, reddish and cold skin, cool feeling of extremities and abdomen, low blood sugar and poor feeding, Hypoxia (low levels of blood oxygen), and heart arrhythmias [4]. Hypothermia can affect good health newborns as well as at-risk newborns. It can happen very quickly in Low Birth Weight (LBW) infants, sick newborns, or newborns that have been resuscitated [5]. Low birth weight (LBW) is defined as a birth weight of a live born infant of less than $2500 \mathrm{~g}$ ( 5 pounds 8 ounces). The use of infant incubators is to prevent excessive heat loss in at-risk newborns or to re-warm and stabilize the temperatures in a newborn that has experienced rapid heat loss. If infant incubators are well maintained, functioning and used properly, incubators can help stabilize and warm infants, particularly LBW infants, and at-risk newborns. The survival of each newborn depends on the ability for it to regulate its body temperature.

Lack of attention to thermoregulation continues to be a cause of unnecessary deaths in the neonatal population. Maintaining a stable body temperature is essential to ensure optimal growth. If the temperature is maintained, caloric expenditure and oxygen consumption would be minimal. Newborn babies, in particular, the preterm and the low birth weight are exquisitely predisposed to hypothermia. No other equipment is identified more with the special care of newborn babies than the warming devices, namely incubators and radiant warmers. When the temperature of the newborn baby fall down to $36.5^{\circ} \mathrm{C}$, we usually use Infant Radiant Warmer (IRW) which mainly includes heat element canopy, mattress and heater, to transfer heat through under radiation which helps the baby to get heat and to raise the temperature into normal value $\left(36.5^{\circ} \mathrm{C}\right.$ $\left.37.2^{\circ} \mathrm{C}\right)$.

It is a common issue that premature babies generally lose heat very quickly than normal babies. The Infant Radiant Warmer is inadvisable for routine care of newborn infants since it can increase insensible water loss and overheat of baby skin. The Infant Radiant Warmer saved the premature infant from hypothermia during the invasive procedure. It is possible to maintain a proper control mechanism (lamp and the distance between the mattress and the heater). In warmer heater is the most important component which is a source for the generation of heat. There is a variety of heaters available on market [500 watts - 600 watts] but the selection of heater is done according to the parameters.

The Neonatal Intensive Care Units (NICUs) often employ Radiant Warmers (RWs) to maintain a stable body temperature in many newborns, as relative to incubators, these devices allow increased visibility and access to the patient. However, some reports suggest RWs predispose infants to a risk of passive overheating. Previous cases have been attributed to manual operational error (for example, in- 
appropriate use of manual control mode, displacement of servo-controlled temperature probe, or malfunctioning units). The present case report details the acute thermometric, cardiovascular, and respiratory responses of a neonate during an accidental bout of passive overheating induced by a fully functional RW in servo control mode used in accordance with standard care procedures.

Infant Radiant Warmer (IRW) often uses to recover hypothermia for newborn babies. In spite of using IRW, some problems still remain due to a lack of knowledge to handle properly the IRW. To control the surrounding temperature the height of the heat source is also an important factor to protect the body temperature of the infant. In the designing of the system has to model the underlying reality of the infant radiant warmer are used for simulation results were similar to reality as possible [6]. The aim of this study is to analyze the heat transfer phenomena from the surrounding area of skin of newborn using Infant Radiant Warmer (IRW). The result can be that the effects of radiation on the skin of newborns using infant radiant warmer treatment. For ensuring accurate temperature for the baby we also want to heat the mattress and the surrounding environment with proper temperature. In brief, our objective is to equip the baby with accurate temperature to remain free from hypothermia. A Finite Element Method was used to simulate the heat and thermal radiation of IRW using the COMSOL Multiphysics software which helps to handle IRW properly to cure hypothermia for a newborn baby.

\section{Mathematical Model}

Heat is generally known as the process of energy transfer. In this study, we have studied the heat transfer efficiency of a new born baby through under infant radiant warmer. In this simulation, the heat conduction equation derived from Fourier's law, stating that the time rate of heat transfer through a material is proportional to the negative gradient of temperature and to the area at right angles, through which the heat is flowing [7].

$$
\boldsymbol{q}=-k \nabla T
$$

The Heat Transfer interfaces define an elliptic partial differential equation for the temperature $T$, of the form [8]:

$$
\rho C_{p} \frac{\partial T}{\partial t}+\nabla \cdot(-k \nabla T)=Q
$$

where $\rho$ is the density of material; $C_{p}$ is the heat capacity of materials; $k$ is the thermal conductivity of material; $Q$ is the volume of heat source; $\boldsymbol{q}$ is the local heat flux, with Dirichlet and Neumann boundary conditions at some boundaries:

$$
-\boldsymbol{n} \cdot(k \nabla T)=0
$$

The Neumann boundary condition is not linear in temperature. A convective cooling condition of type keeps the linearity of the problem when the heat transfer coefficient $h$ is constant [9].

$$
-\boldsymbol{n} \cdot(k \nabla T)=h\left(T_{e x t}-T\right)
$$


A radiative condition of type

$$
-\boldsymbol{n} \cdot(k \nabla T)=\varepsilon \sigma\left(T_{a m b}^{4}-T^{4}\right)
$$

Convective heat flux is a flux depending on the temperature difference between the body and the adjacent fluid [10]. The convective heat flux equation

$$
q_{0}=h\left(T_{\text {ext }}-T\right)
$$

where $T_{\text {ext }}$ is external temperature, $\varepsilon$ is the surface emissivity, $\sigma$ is the Stefan-Boltzmann constant.

The importance radiation heat transfer in any thermal system analysis is not only in determining surface radiation energy but also the radiation exchange between the surface and other surface in its surrounding. When a hot surface is radiating to its cooler surrounding, the total radiation heat loss can be calculated from

$$
P=\varepsilon \sigma A\left(T_{0}^{4}-T^{4}\right)
$$

where $T_{0}[\mathrm{~K}]$ is the temperature of the surface; $T[\mathrm{~K}]$ is the temperature of surroundings and $\varepsilon$ is emissivity, coefficient defining how efficiently surface emits energy relative to the blackbody [11].

The net inward heat flux due to surface-to-ambient radiation is

$$
-\boldsymbol{n} \cdot \boldsymbol{q}=\varepsilon \sigma\left(T_{a m b}^{4}-T^{4}\right)
$$

Diffuse surfaces reflect radiative intensity uniformly in all directions. A radiative heat source contribution

$$
q=\varepsilon\left(G-e_{b}(T)\right)
$$

where, $e_{b}(T)$ is the blackbody hemispherical total emissive power, $G$ is the irradiation.

Total heat source of the volume is

$$
Q=\frac{P_{t o t}}{V}
$$

where, $Q$ is the total volume of heat source, $V$ is total volume in the selected domain.

\section{Computational Domain and Mesh Design}

The geometry of a real baby and radiant warmer is very complex and detailed in Figure 1. For the purposes of this study a simplified model has been built subdomain of CAD. The Whole domain consists of four main parts. The four parts are containing heating element canopy, heater, new born baby and mattress. Use the size of machine at present for received the right information of thermal radiation analysis to design the infant radiant warmer to be equal to real size at hospitals. In Figure 2 and Figure 3, the design of heating element canopy have semicircle character with a radius of $20 \mathrm{~cm}, 2 \mathrm{~cm}$ thick and $40 \mathrm{~cm}$ long and leave a space from newborn mattress 70 centimeters. The size of the infrared light designed is a cylinder of radius $1 \mathrm{~cm}$ and length $40 \mathrm{~cm}$ instead of the real baby geo- 
metry whose area is $0.1106 \mathrm{~m}^{2}$. The whole computational domain of the infant radiant warmer is given in the Figure 4.

We have to confirm that our mathematical and computational approach is appropriate and accurate. The best way to gain this confidence is to start the modeling with something simple, both in terms of the geometry mesh and boundary conditions. Such a model can be used to compute various simulations with different case setups in order to find the effects of the physical behavior on the results. Therefore the set of results presented in this study is used for observing the behavior of the natural convection phenomena combined with a radiant heat source.

As the mesh design plays a significant role in accuracy of the numerical results, in this simulation, the complete mesh consist of 5228 elements in tetrahedral shape with 5118 elements and triangular shape with 338 elements in Figure 5.

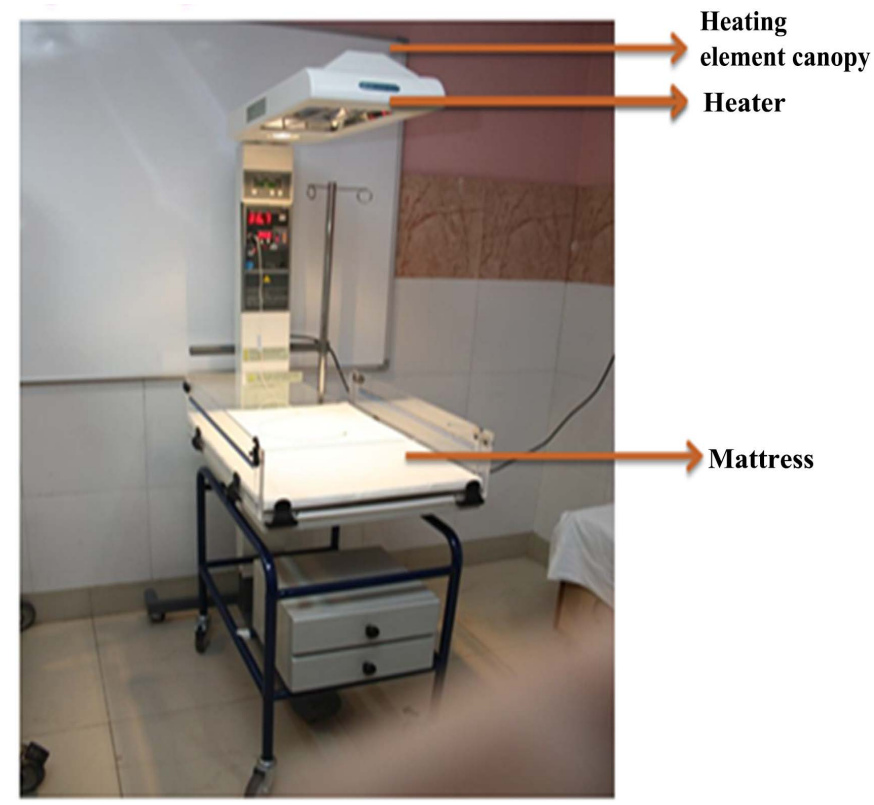

Figure 1. Infant radiant warmer (IRW).

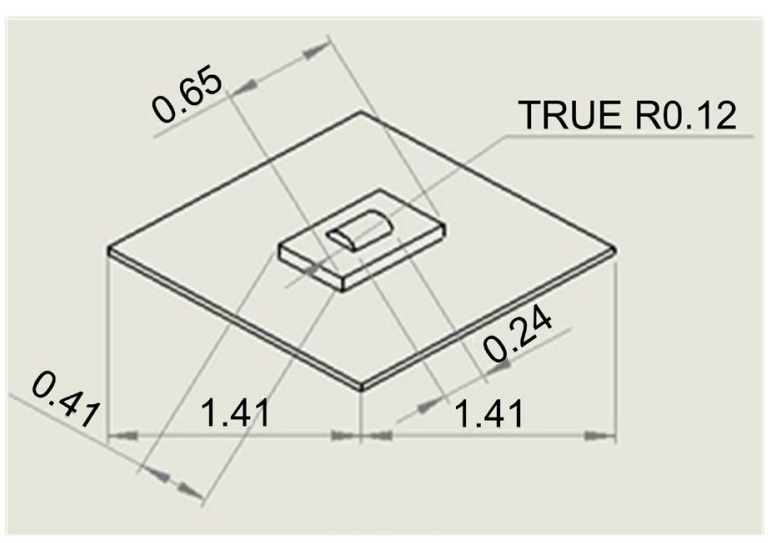

Figure 2. Computational domain with the dimensions of the mattress. 


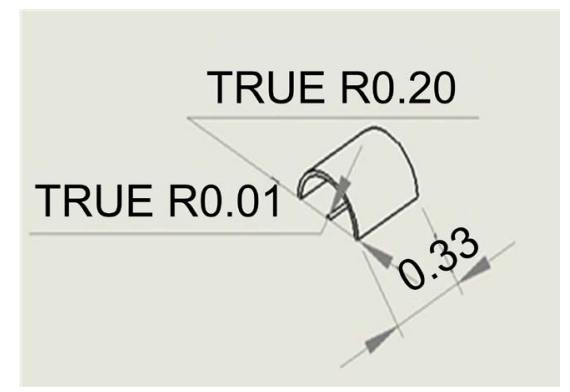

Figure 3. Dimensions of the eater and heating element canopy.

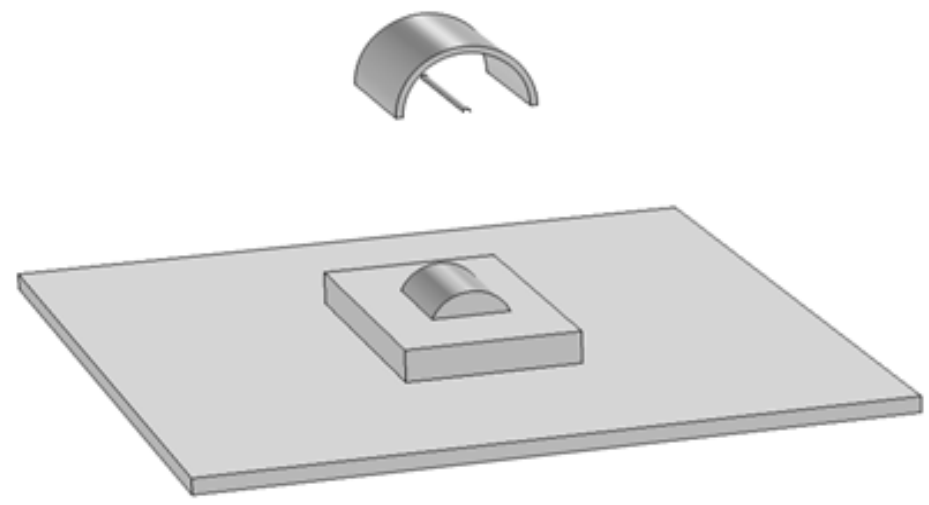

Figure 4. Computational domain of the entire infant radiant warmer.

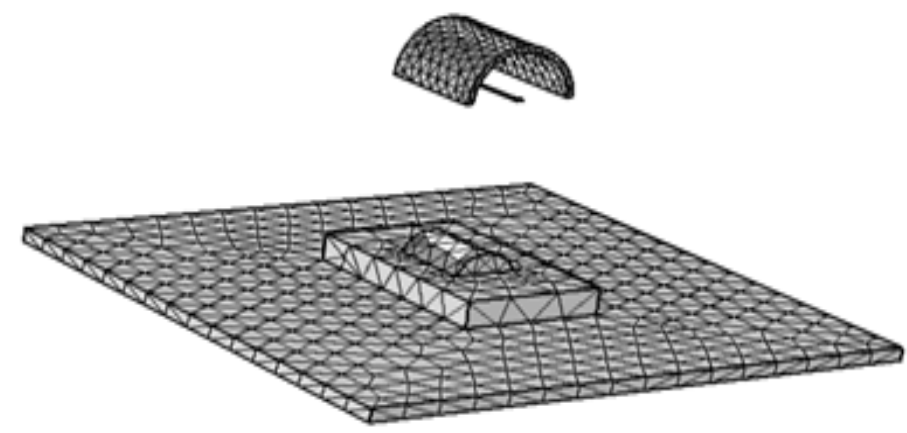

Figure 5. The mesh design for the computational domain.

\section{Simulation Results and Discussions}

We use COMSOL Multiphysics (version 4.2a) solver to implement finite element analysis and carried out the simulation for an hour. Simulation module was selected on heat transfer module with a heat transfer in solids and surface to surface radiation (ht) according to an industrial, scientific and medical (ISM) radio bands criteria. The model assumes that this physical system is dominated by radiation and convection cooling. The convective cooling of the mattress and heating element canopy to the air (at $301^{\circ} \mathrm{K}$ ) is modeled using a heat transfer coefficient, $h$ (in this model set to $500 \mathrm{~W} /\left(\mathrm{m}^{2} \mathrm{~K}\right)$. The model simulates the lamp as a solid object with a volume heat source of $500 \mathrm{~W}$. It is insulated on all surfaces except for the bottom, which faces the mattress. At this surface, heat leaves the 
lamp as radiation only. In order to capture the lamp's transient startup time, the model uses a low heat capacity, $C_{P}$ for the solid $10 \mathrm{~J} /(\mathrm{kg} \cdot \mathrm{K})$. The lamp's other thermal properties are identical to those of copper metal.

In this case, we assume that the mattress dissipates energy via radiation and convection on all surfaces. The heating element canopy is insulated at all surfaces except the top, which is subjected to both convection and radiation. The thermal material properties are set to those of alumina. In this part we discuss heat transfer radiation for different watts. In Table 1, we have provided the measurement of mattress, Heater and heating element etc., while Table 2 represents the parameter values considered for our simulation.

We run our simulation for one hour. The distribution of temperature and radiosity are shown in Figure 6 and Figure 7 respectively when the heater at 500

Table 1. Specifications of model designed for infant radiant warmer system.

\begin{tabular}{cc}
\hline Modeling Object & Size $(\mathbf{c m})$ \\
\hline Mattress & $50 \times 80 \times 10$ \\
Heating element canopy & Diameter $20 \times 40$ \\
Heating element canopy layer & 2 \\
Heater & Diameter $1 \times 40$ \\
Distance of mattress and heater & 70 \\
\hline
\end{tabular}

Table 2. Parameters values used for the simulation [12].

\begin{tabular}{cccc}
\hline Material & Thermal Conductivity & Density & Heat capacity \\
\hline Heater & $400 \mathrm{~W} / \mathrm{mK}$ & $8700 \mathrm{~kg} / \mathrm{m}^{3}$ & $10 \mathrm{~J} / \mathrm{kgK}$ \\
Heating element canopy & $155 \mathrm{~W} / \mathrm{mK}$ & $2730 \mathrm{~kg} / \mathrm{m}^{3}$ & $893 \mathrm{~J} / \mathrm{kgK}$ \\
Mattress & $27 \mathrm{~W} / \mathrm{mK}$ & $2000 \mathrm{~kg} / \mathrm{m}^{3}$ & $500 \mathrm{~J} / \mathrm{kgK}$ \\
\hline
\end{tabular}

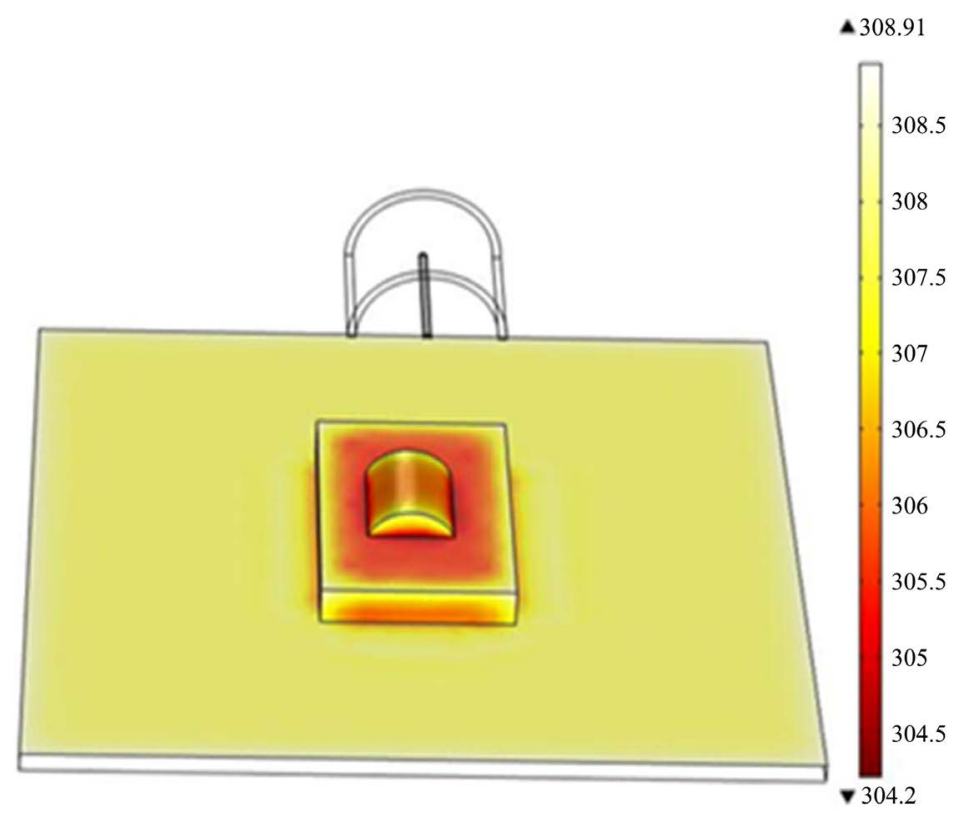

Figure 6. Surface temperature for the computational domain for 500 watts. 


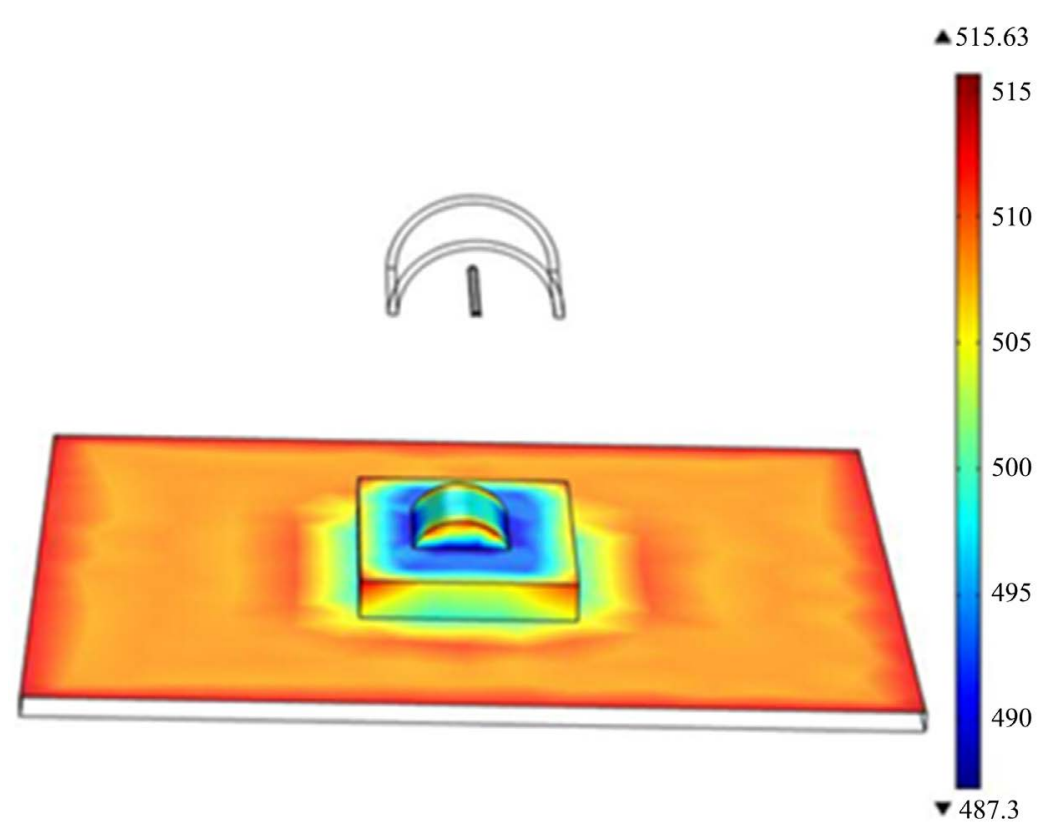

Figure 7. Surface radiosity for the computational domain for 500 watts.

watts. In Figure 6, the temperature distribution rate is taken from the heater in the simulation period. It shows that the temperature of the mattress raises up to 33.5 - 34 degrees Celsius and skin temperature raises up to 34.5 - 35 degrees Celsius. Figure 7 presents the surface radiosity that received thermal radiation from the heater that shows power value at surface of materials near the heater. A 500 watts heat source is used in the heater which diffuses heat to the newborn baby. It is found that the baby domain gets heat from the heater and also absorbs heat from the mattress. Through this process, the temperature condition is now improved as needed to the newborn and maintains the regular temperature to overcome hypothermia.

Figure 8 shows that the baby's different arm temperature range from 304 308 degree Celsius. In which indicates that the surface gains heat rather than releasing it to the surrounding air. It has been observed that within the obtained results there is good qualitative agreement with the experimental data. For example, the average temperature of the baby skin obtained from the experiments and the numerical results are very close.

Similarly Simulation results of this study are shown in Figure 9 and Figure 10 when the heater uses a bulb of 600 watts. It is observed that the temperature of mattress raises up to $36-36.5$ degrees Celsius and skin temperature raises up to 37 - 37.5 degrees Celsius.

Figure 11 shows that the baby's different arm temperature range from 308 312 degree Celsius. It has been observed that skin temperature of a baby above 39 degree Celsius which shows a disagreement with the medical data. Figure 12 shows a comparison of temperature distribution at two times at thirty minutes and sixty minutes after simulation. It is found that the temperature have gradually been increasing with respect to time. 


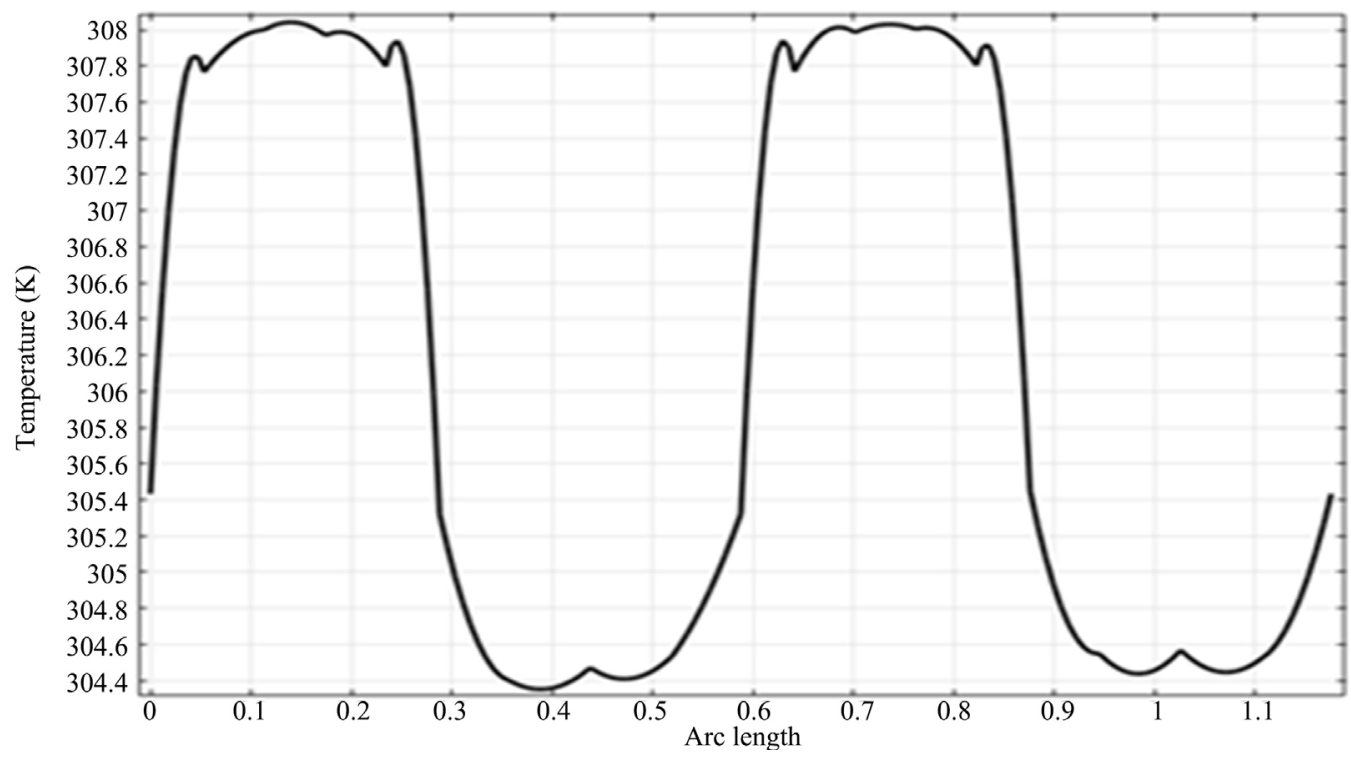

Figure 8. Temperature of edges of the baby domain for 500 watts.

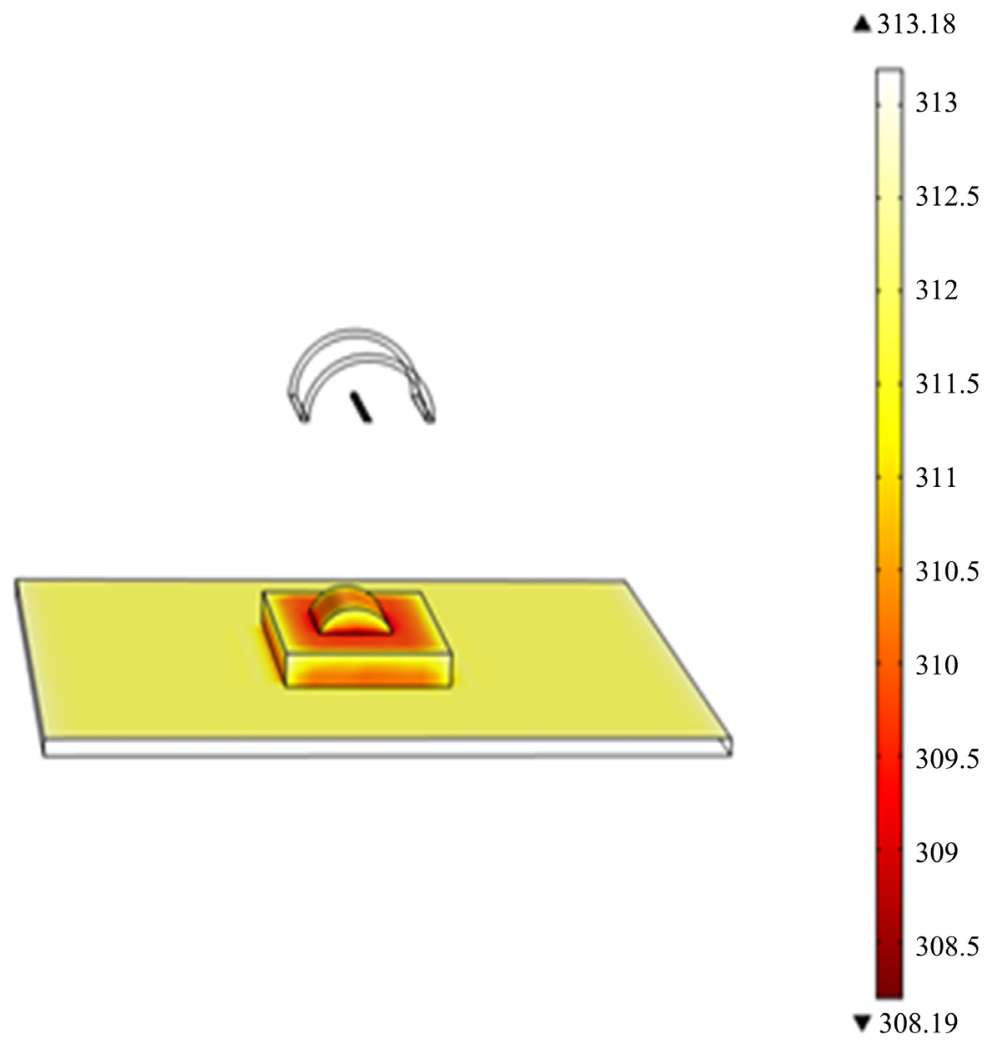

Figure 9. Surface temperature for the computational domain for 600 watts.

Figure 13 presents the temperature distribution by line graphs of three different times. As we mentioned earlier the simulation carried out for one hour, so we have taken time interval is 20 minutes. It is also found that with respect to time the temperature of the baby body is increasing slowly.

From the comparison of two different watts of bulbs 500 watts and 600 watts 

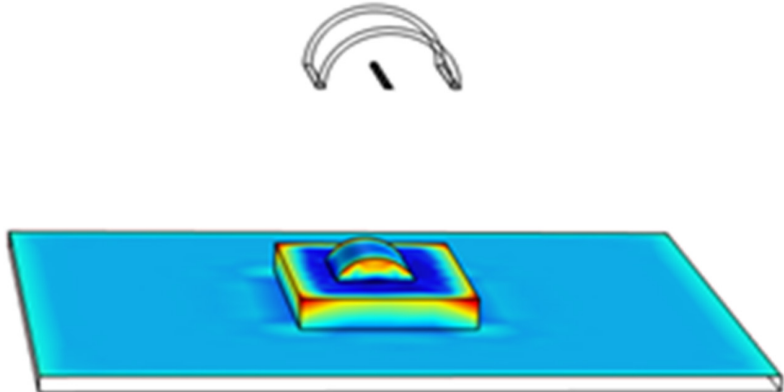

Figure 10. The surface radiosity for the computational domain for 600 watts.

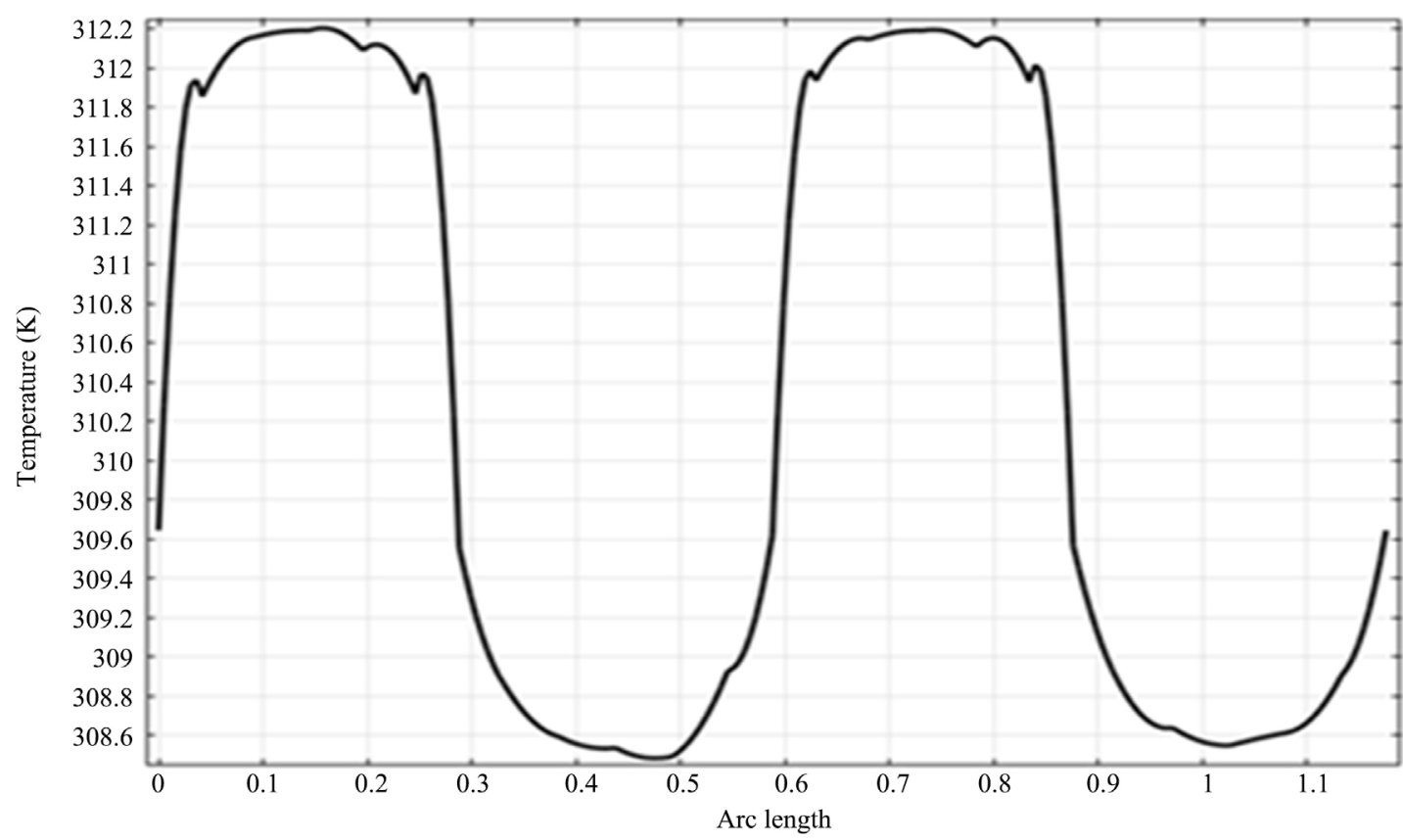

Figure 11. Temperature of edges of the baby domain for 600 watts.

respectively in Figure 6 and Figure 9, it is found that bulb with 500-watts provide better results than that obtained using 600 watt bulb in an incubator. For a 600-watts bulb may cause skin damage for the baby according to the temperature reading from the simulation. With the help of the proposed model, it is 


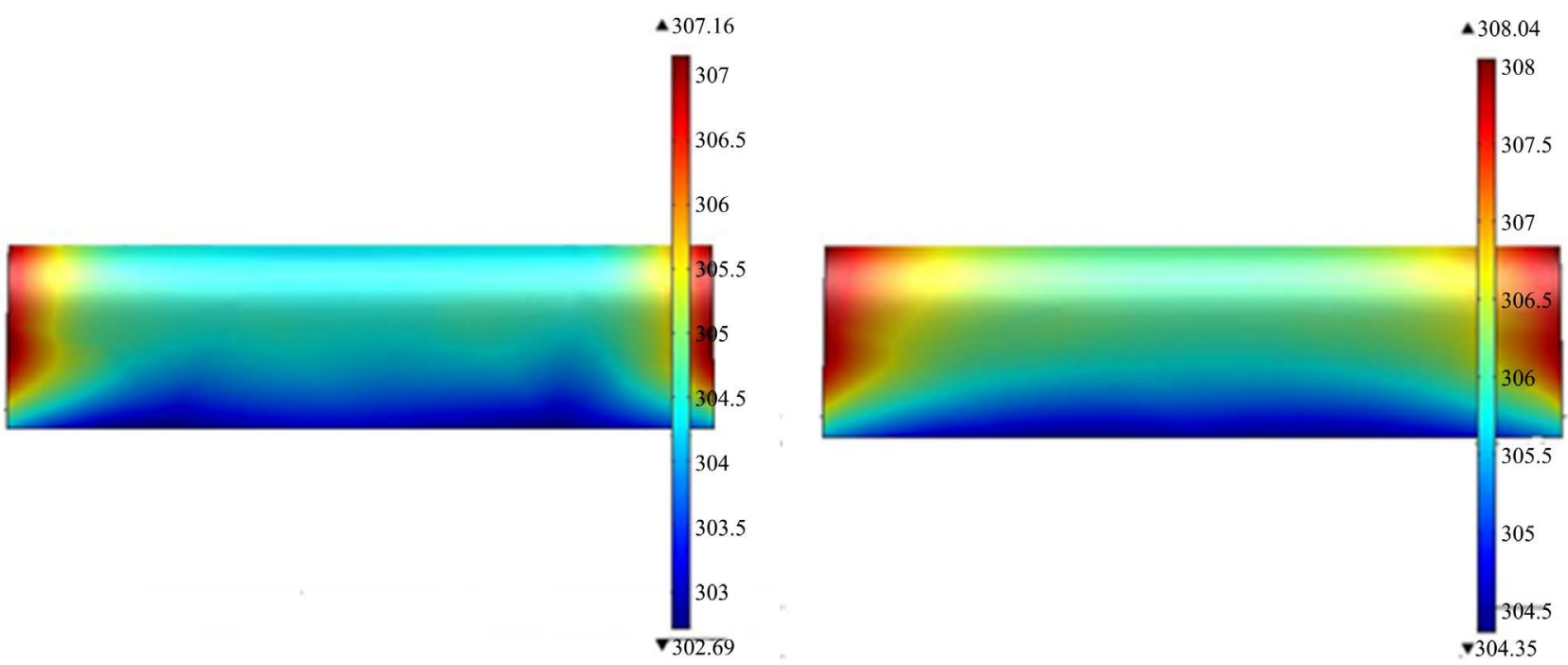

Figure 12. Temperature of the baby domain for 500 watts at $30 \mathrm{~min}$ and $60 \mathrm{~min}$ time.

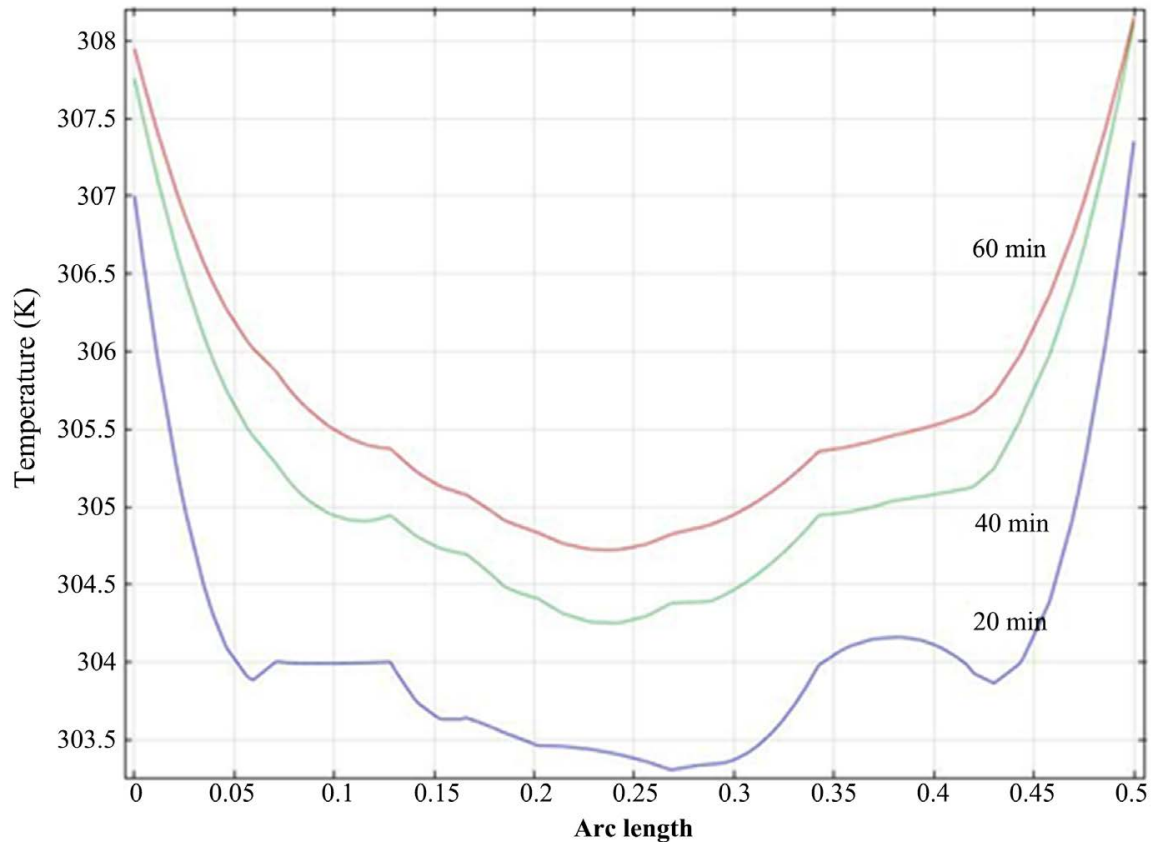

Figure 13. Temperature line graph of the baby domain for 500 watts at three different times.

suggested to treat hypothermia by using 500 watts in heater for an Infant Radiant Warmer. In this simulation, it is observed that while the heat source of 500 Watts is emitted for 30 minute radiation time, Infant area temperature increases about 34.5 - 35 degree Celsius and mattress area about 33.5 - 34 degree Celsius respectively. It should be noted that the simulation results were similar to the literature of Medical Science. The results of thermal radiation from the infrared lamp to the mattress domain by using the three-dimensional finite element methods simulation is also as close to the true results. The domain of the baby gets the proper temperature through the surface of the mattress. 


\section{Conclusion}

A computational model for heat transfer analysis using the Finite Element Method is developed to investigate the heat transfer phenomenon for an Infant Radiant Warmer that uses to regulate the body temperature of a newborn baby in case of hypothermia. The defuse temperature related to heat transfer from Infant Radiant Warmer is simulated to study the best range of light source to overcome the hypothermia. For our simulation, we considered the distance between the infant's mattress and the bottom surface of the heater unit as $70 \mathrm{~cm}$ and the heater power 500 watts, and 600 watts bulbs. It is found that the 500 watts heater bulb provides better results than that obtained using a 600-watt bulb in an incubator. It has been observed that the temperature of the mattress raises up to 3636.5 degrees Celsius and skin temperature raises up to 37 - 37.5 degrees Celsius. A gradual temperature rise of the baby's body domain with rest to time is observed. The obtained results have shown a good qualitative agreement with the experimental data. It is also found that the average temperature of the mattress obtained from the experiments and the numerical results are very close. The thermal radiation from the infant radiant warmer diffuses heat to the mattress consequently to the baby's body domain.

\section{Acknowledgements}

The authors gratefully acknowledge the technical support to The Centre of excellence in Mathematics, Department of Mathematics, Mahidol University, and Bangkok, Thailand and to the Simulation Lab, Department of Mathematics, Chittagong University of Engineering and Technology, Bangladesh, for the support to complete this research.

\section{Conflicts of Interest}

The authors declare no conflicts of interest regarding the publication of this paper.

\section{References}

[1] Hypothermia. http://www.webmd.com/a-to-z-guides/what

[2] Jirapaet, K. and Jirapaet, V. (1991) Rewarming Hypothermic Infant with Siriraj Radiant Warmer Model-2. Department of pediatrics, Faculty of medicine Siriraj Hospital, Mahidol University, Bangkok, 43, 299-305.

[3] World Health Organization. Maternal Health and Safe Motherhood Programme and Meeting of Technical Working Group on Thermal Control of the Newborn (1992: Geneva, Switzerland) (1993) Thermal Control of the Newborn: A Practical Guide. World Health Organization, Geneva, Switzerland.

[4] http://www.medicinenet.com/hypothermia_extended_exposure_to_cold/page3.htm

[5] https://path.azureedge.net/media/documents/TS_newborn_thermal_rpt.pdf

[6] Roongprasert, K., Phasukkit, P., Pintavirooj, C., et al. (2012) Heat Transfer Efficiency Analysis of Infant Radiant Warmer by 3D Finite Element Method. The 5 th 2012 Biomedical Engineering International Conference, Muang, Thailand, 5-7 De- 
cember 2012, 1-4.

[7] Liu, I-S. (1990) On Fourier's Law of Heat Conduction. Continuum Mechanics and Thermodynamics, 2, 301-305. https://doi.org/10.1007/BF01129123

[8] Al Mamun, A., et al. (2018) A Study on an Analytic Solution 1D Heat Equation of a Parabolic Partial Differential Equation and Implement in Computer Programming. International Journal of Scientific and Engineering Research, 9, 913-921.

[9] Heat Transfer Module. https://cn.comsol.com/

[10] Kurazumi, Y., Tsuchikawa, T. and Ishii, J. (2008) Radiative and Convective Heat Transfer Coefficients of the Human Body in Natural Convection. Building and Environment, 43, 2142-2153. https://doi.org/10.1016/j.buildenv.2007.12.012

[11] Tam, W.C., Yuen, W.W. and Chow, W.K. (2015) Numerical Study on the Importance of Radiative Heat Transferin Building Energy Simulation. Numerical Heat Transfer, Part A: Applications, 69, 694-709.

https://doi.org/10.1080/10407782.2015.1090822

[12] Basutkar, Y.S. and Hippargi, S.S. (2019) Reliable Infant Warmer to Avoid Accidental Overhead of Newborn. International Journal of Research and Scientific Innovation, 6, 98-102. 